A number of epidemiological investigations have identified asthma prevalence in cleaners around 1.5-2.0 times those of reference populations. There are around 700,000 cleaners in the $\mathrm{UK}$, asthma prevalence is around $8 \%$, and that suggests a high burden of work-related disease. However, a clinical diagnosis of occupational asthma in cleaners is established relatively rarely. We have investigated the hypothesis that this discrepancy occurs because cleaner's asthma is a form of low dose irritant asthma that is visible to epidemiologists but does not have the typical clinical features of occupational asthma. A questionnaire was sent to 1400 cleaners working in local hospitals and universities. $14 \%$ had a previous diagnosis of asthma, and in $32 \%$ of these the asthma started after they began work as a cleaner. Investigations for possible occupational asthma comprised paired measurements of airway responsiveness at and away from work $(\mathrm{n}=13)$, serial PEF analysed using OASYS-2 $(\mathrm{n}=13)$ and a structured clinical history $(\mathrm{n}=10) .5$ subjects had a greater than 3 fold improvement in PD20 away from work, and 2 subjects had OASYS score $>2.5$ indicating a probable occupational effect. 1 subject had both. The clinical histories were sent to 9 physicians with an interest in occupational asthma who were asked to score them for the likelihood of occupational asthma on a scale 0 to $100 \%$ with and without the OASYS scores and the airway responsiveness measurements. Before seeing the investigation results, 7 of the 90 individual scores ( 9 physicians $\mathrm{x} 10$ subjects) were above $50 \%$ indicating that the diagnosis of occupational asthma was thought likely. After seeing the investigation results, 29 of the 90 scores were above 50\%. The mean probability score based on the history alone did not exceed $50 \%$ for any cleaner but was above $50 \%$ for 2 cleaners when the investigations were taken into account. These findings support the view that cleaner's asthma has features that make it difficult to identify from the clinical history.

\section{P133 LONGITUDINAL DECLINE IN FEV1 IN OCCUPATIONAL ASTHMA DUE TO IRRITANTS IS NOT ALTERED BY REMOVAL FROM EXPOSURE}

${ }^{1} E$ Butterfield, ' $\mathrm{GI}$ Walters, 'VC Moore, ${ }^{2} \mathrm{P}$ Nightingale, ${ }^{1} \mathrm{~A}$ Turner, ${ }^{1} \mathrm{PS}$ Burge. ${ }^{1} \mathrm{Heart}$ of England NHS Foundation Trust, Birmingham, UK; '2University of Birmingham, Birmingham, UK

\subsection{6/thoraxjnl-2014-206260.274}

Introduction Airborne irritants and allergens in the workplace can induce and trigger occupational asthma (OA). OA due to sensitisation is associated with an accelerated rate of decline in $\mathrm{FEV}_{1}(100 \mathrm{mls} / \mathrm{yr})$ compared with healthy controls $(25 \mathrm{mls} / \mathrm{yr})^{1}$, which improves on removal from exposure. We sought to describe the rate of $\mathrm{FEV}_{1}$ decline in patients with irritantinduced OA before and after their removal from exposure.

Methods Cases of irritant-induced OA reported between 1991 and 2011 were identified from the SHIELD database (a voluntary reporting scheme for OA) and their demographic characteristics and serial $\mathrm{FEV}_{1}$ measurements were gathered. Generalised estimating equations with an exchangeable correlation structure were used to calculate an average rate of $\mathrm{FEV}_{1}$ decline for all patients before and after removal from exposure.

Results A total of $526 \mathrm{FEV}_{1}$ readings (179 prior to removal, 347 post removal) were gathered from 52 patients. 30 patients had $\mathrm{FEV}_{1}$ data both before and after removal; 5 patients had $\mathrm{FEV}_{1}$ data only before removal, and 17 patients had $\mathrm{FEV}_{1}$ data only after removal; demographics were not significantly different between the groups. $\mathrm{FEV}_{1}$ decline prior to removal of the irritant was $44 \mathrm{ml} /$ year (95\% CI: 32-58) and $\mathrm{FEV}_{1}$ decline after removal was $49 \mathrm{ml} /$ year (95\% CI: 36-62). There was no significant difference between the intercepts of the two lines, implying no improvement in $\mathrm{FEV}_{1}$ after removal from exposure.

Conclusion In this cohort, irritant-induced OA was associated with an accelerated decline in $\mathrm{FEV}_{1}$, which persisted after removal from the irritant. These results might be attributed to differences in the underlying pathology of sensitisation and irritant-induced OA, differences in patient behaviour, or differences in treatments offered to the two groups.

\section{REFERENCE}

1 Anees $\mathrm{W}$ et al. FEV 1 decline in occupational asthma. Thorax 2006;61:751-5

\section{P134 SENSITISATION TO CROSS-REACTIVE CARBOHYDRATE DETERMINANTS IN BRITAIN'S BAKERS: THE IMPLICATIONS FOR HEALTH SURVEILLANCE}

${ }^{1} \mathrm{H}$ Harrison, ${ }^{1} \mathrm{~J}$ Welch, ${ }^{1} \mathrm{~S}$ Schofield, ${ }^{2} \mathrm{~J}$ Cannon, ${ }^{2} \mathrm{~B}$ Fitzgerald, ${ }^{1} \mathrm{M}$ Jones. ${ }^{1} / \mathrm{mperial}$ College, London, UK; ${ }^{2}$ Royal Brompton and Harefield NHS Trust, London, UK

\subsection{6/thoraxjn-2014-206260.275}

Introduction and objectives The diagnosis of baker's asthma as part of health surveillance schemes in some UK supermarkets relies on determining sensitisation to wheat flour and/or alpha amylase. Recently, data have emerged suggesting that serum $\operatorname{IgE}$ analysis in bakers may be complicated by the presence of clinically irrelevant specific IgE to cross-reactive carbohydrate determinants (CCDs), which are complex-type Asn (N)-linked glycan structures commonly formed in plants. Potentially this might lead to false positive flour specific IgE assays, which would have an impact on bakers undergoing surveillance. The aim of this study was to identify the prevalence of CCD sensitisation in UK bakers and investigate the impact of CCD specific IgE within a health surveillance setting.

Methods Serum samples from UK bakers attending our occupational asthma clinic $(\mathrm{n}=209)$ were analysed for specific IgE to CCD $\left(\mathrm{MUXF}^{3}\right.$ ) using ImmunoCAP assay (Phadia). Any positive samples were further tested for specific IgE to grass pollen, and competitive inhibition assays were used to determine cross-reactivity between CCD, flour and grass pollen.

Results Sensitisation rates to CCD in our population of UK bakers were low (7\%) despite high sensitisation rates to grass pollen $(48 \%)$ and flour (60\%). Sensitisation to CCD was more prevalent in those sensitised to either flour or grass than in those not sensitised to flour $(11.5 \%$ vs $0 \%, \mathrm{p}<0.001)$ or grass $(10.9 \%$ vs $2.8 \%, \mathrm{p}=0.025)$. We observed cross reactivity between flour and grass pollen and competitive inhibition assays between CCD and flour or grass pollen revealed cross-reactivity in some but not all sensitised bakers.

Conclusions Our study demonstrated that a minority of bakers were sensitised to CCD and, interestingly, this was associated with being co-sensitised with both flour and grass. It is unlikely that CCDs have major implications for the health surveillance for UK bakeries. In the minority of bakers with CCD specific $\mathrm{IgE}$, there was some suggestion that CCDs may play a role in the cross-reactivity between flour and grass pollen, although in others it was less likely. Within the clinical setting, it may be prudent to measure CCD specific IgE in bakers who are co-sensitised to both flour and grass pollen. 